\title{
Applicability of 3-Dimensional Quantitative Coronary Angiography-Derived Computed Fractional Flow Reserve for Intermediate Coronary Stenosis
}

\author{
Kyoichiro Yazaki, MD; Masato Otsuka, MD, PhD; Shohei Kataoka, MD; \\ Mitsuru Kahata, MD; Asako Kumagai, MD; Koji Inoue, MD, PhD; \\ Hiroshi Koganei, MD, PhD; Kenji Enta, MD, PhD; Yasuhiro Ishii, MD, PhD
}

Background: Quantitative flow ratio (QFR) is a newly developed image-based index for estimating fractional flow reserve (FFR).

Methods and Results: We analyzed 151 coronary arteries with intermediate stenosis in 142 patients undergoing wire-based FFR measurement using dedicated software. Predefined contrast flow QFR, which was derived from 3-dimensional quantitative coronary angiography (3-D QCA) withThrombolysis in Myocardial Infarction (TIMI) frame counts, was compared with FFR as a reference. QFR had good correlation $(r=0.80, P<0.0001)$ and agreement (mean difference: $0.01 \pm 0.05)$ with FFR. After applying the FFR cut-off $\leq 0.8$, the overall accuracy rate of $Q F R \leq 0.8$ was $88.0 \%$. On receiver operating characteristics analysis, the area under the curve was 0.93 for QFR. In contrast, 3-D QCA-derived anatomical indices had insufficient correlation with FFR and diagnostic performance compared with QFR.

Conclusions: QFR had good correlation and agreement with FFR and high diagnostic performance in the evaluation of intermediate coronary stenosis, suggesting that QFR may be an alternative tool for estimating myocardial ischemia.

Key Words: 3-dimensional quantitative coronary angiography; Fractional flow reserve; Myocardial ischemia; Quantitative flow ratio

$\mathbf{P}$ recise assessment of coronary artery disease (CAD), especially intermediate stenosis, is essential for the estimation of myocardial ischemia. Anatomic severity on coronary angiography $(\mathrm{CAG})$ is limited in the assessment of functional severity.1,2 Pressure-derived fractional flow reserve (FFR) for the assessment of myocardial ischemia has been established because of its availability and feasibility. Moreover, percutaneous coronary intervention based on FFR guidance has good prognostic accuracy in intermediate coronary stenosis. ${ }^{36}$ Wire-based FFR measurement, however, has several problems: (1) the hyperemic state is required, causing chest discomfort; (2) adenosine triphosphate, which produces the hyperemic state, cannot be used in patients with severe asthma, hypotension, or atrioventricular conduction disturbances; and (3) it is challenging to deliver the pressure wire in tortuous coronary arteries with complex anatomy. Recently, there have been several reports on the usefulness of image-based FFR with computational fluid dynamics (CFD). ${ }^{7-11}$ Notably, non-invasive FFR derived from computed tomography CAG (CT-FFR) along with CFD had better diagnostic performance compared with the standard anatomical index-based CT CAG. ${ }^{912-14}$ As another image-based approach to FFR, computed FFR using CAG data has been introduced.7,10,11,15
Tu et al reported good diagnostic performance of FFR based on 3-dimensional quantitative CAG (3-D QCA) and Thrombolysis in Myocardial Infarction (TIMI) frame counts. ${ }^{11}$ The 3-D QCA-based FFR was called quantitative flow ratio (QFR), which involves an advanced algorithm that enables fast computation of pressure decreases in intermediate $\mathrm{CAD}$ without any invasive physiologic measurements or pharmacologic hyperemia induction. The international, multicenter FAVOR pilot study confirmed the good diagnostic performance of QFR and its designated software. ${ }^{15}$ Contrast-flow QFR, which is based on the computational hyperemic state and TIMI frame count, had good correlation and agreement with FFR and similar diagnostic accuracy to that achieved using the pharmacologic hyperemic state.

The aim of this study was therefore to confirm the applicability of QFR, using wire-based FFR as the reference standard in the present single-center cohort.

\section{Methods}

\section{Study Design}

The present study was a retrospective, single-center, observational study. Patients who underwent CAG and FFR

Received December 11, 2016; revised manuscript received February 10, 2017; accepted February 13, 2017; released online March 22, 2017 Time for primary review: 25 days

Department of Cardiology, Cardiovascular Center, Ogikubo Hospital, Tokyo, Japan

Mailing address: Kyoichiro Yazaki, MD, Department of Cardiology, Cardiovascular Center, Ogikubo Hospital, 3-1-24 Imagawa, Suginami-ku, Tokyo 167-0035, Japan. E-mail: kamisamakaranookurimono@gmail.com

ISSN-1346-9843 All rights are reserved to the Japanese Circulation Society. For permissions, please e-mail: cj@j-circ.or.jp 


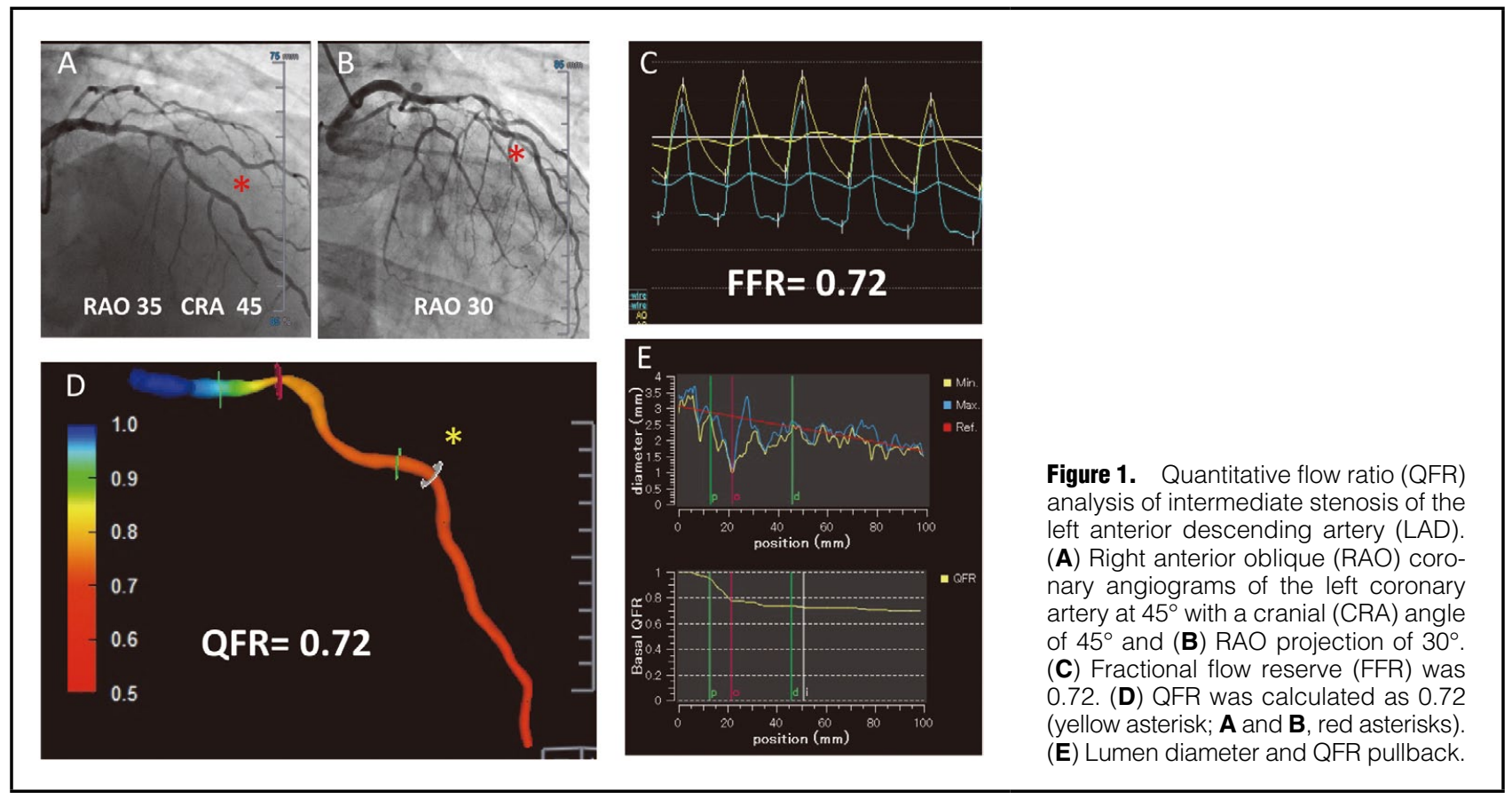

measurements were enrolled. 3-D QCA with QFR reconstructed from CAG was analyzed and compared with FFR as a reference in a core laboratory at the same institute. This study was conducted after receiving ethics approval of the institutional review board. All patients provided written informed consent before enrollment.

\section{Subjects and Target Lesions}

One hundred and seventy-one consecutive vessels in 156 patients who underwent CAG and subsequent FFR measurement between May 2014 and July 2016 were screened. Exclusion criteria were as follows: (1) lack of 2 optimal angiographic projections at least $25^{\circ}$ apart; (2) overlapping interrogated vessels with too much shortening without preferred references in proximal or distal vessels; (3) insufficient injected contrast for QCA analysis; and (4) location of the target lesion at the ostium of the left or right coronary artery.

\section{3-D QCA Reconstructed With Angiography}

CAG was performed using the monoplane or biplane X-ray system (Infinix Celeve-i INFX-8000V/8000C; Toshiba Medical Systems, Tokyo, Japan). These images were recorded at 15 frames/s. The 3-D images were reconstructed using validated software (QAngio ${ }^{\circledR} \mathrm{XA} / 3-\mathrm{D}$; Medis, Leiden, the Netherlands) by selected investigators who were blinded to FFR. Initially, we selected 2 angiographic projections $>25^{\circ}$ apart (Figure 1A,B). Subsequently, we registered proximal and distal points as the references and the regions of interest. The 3-D QCA was reconstructed with the proposed analytic model, which was based on geometrical features derived from the entrance angle of coronary stenosis, angularity of the center line, and reference points of the lumen diameter. The percent diameter stenosis (DS $\%$ ), lesion length, minimum lumen diameter (MLD), reference proximal and distal vessel diameters, and percent area stenosis ( $\mathrm{AS} \%$ ) were calculated using 3-D QCA.

\section{Wire-Based FFR}

FFR was measured in all patients using a coronary pressure wire (Aeris ${ }^{\mathrm{TM}}$; St. Jude Medical, St. Paul, MN, USA; and Verrata $^{\circledR}$; Volcano, San Diego, CA, USA). After calibration and equalization, the pressure wire was advanced distally to the stenosis. Maximum hyperemia was induced with i.v. adenosine triphosphate at a concentration of $150-180 \mu \mathrm{g} /$ $\mathrm{kg} / \mathrm{min}$. Both the distal coronary pressure at the pressure sensor and the proximal pressure at the end-hole of the catheter were recorded simultaneously. The pressure sensor was pulled back to the catheter tip to check or correct the pressure drift (Figure 1C).

\section{Calculation of QFR}

Following 3-D QCA reconstruction, QFR was calculated using the same software. QFR was based on the following principles: (1) coronary pressure does not decrease unless stenosis exists; ${ }^{\mathbf{1 6}}$ (2) coronary flow determines the pressure decrease using the fluid dynamics equation; ${ }^{17}$ (3) stenosis characteristics are determined by the deviation of the stenosis lesion with respect to the reference size; and (4) mass flow rate depends on the mean flow velocity and reference size at each point. In the present study, the contrast-flow QFR pullback was adopted: TIMI frame count was measured on CAG and the coronary flow velocity (CFV) was used as the modeled hyperemic flow velocity (HFV).

The relationship between $\mathrm{HFV}$ and CFV has been reported previously using several equations. After calculating $\mathrm{HFV}$, integration of the pressure decrease of all subsegments proximal to that interrogated location results in the QFR at the arbitrary position. The detailed algorithm and theory of QFR computation have been described in a previous report. ${ }^{15}$ Examples of reconstructed 3-D QCA and measured QFR are shown in Figure 1D. Lumen diameter pullback and QFR are shown in Figure 1E.

\section{Measurement and Analysis}

Wire-based FFR and QFR were analyzed and validated in 
the core laboratory of the present institute. QFR calculation was performed by the selected investigators who were blinded to the results of wire-based FFR.

\section{Statistical Analysis}

Normally distributed continuous variables are expressed as mean $\pm \mathrm{SD}$ and non-normal variables as median (IQR). Categorical variables are expressed as percentages. Data were analyzed on a per-patient basis for clinical characteristics and on a per-vessel basis for the remaining calculations. Pearson correlation was used to quantify the correlation between FFR and QFR; Spearman correlation was used to quantify the correlations between FFR and indices of 3-D QCA. Agreement between FFR and QFR was assessed on Bland-Altman plot. The performance of QFR for predicting functionally significant stenosis was assessed using sensitivity, specificity, positive predictive value (PPV), negative predictive value (NPV), and diagnostic accuracy. The area under the curve (AUC) on receiver operating characteristic (ROC) analysis was used to assess the diagnostic accuracy of QFR and 3-D QCA.

\begin{tabular}{|c|c|}
\hline \multicolumn{2}{|c|}{ Characteristics } \\
\hline Mean age (years) & $72.5 \pm 9.5$ \\
\hline Male & $100(70.4)$ \\
\hline Mean BMI (kg/m²) & $23.9 \pm 3.2$ \\
\hline Current smoking & $33(23.2)$ \\
\hline Hypertension & $101(71.1)$ \\
\hline Hyperlipidemia & $88(62.0)$ \\
\hline Diabetes & $41(28.9)$ \\
\hline \multicolumn{2}{|l|}{ Cardiovascular history } \\
\hline Prior $\mathrm{PCl}$ & $58(40.8)$ \\
\hline Prior CABG & $2(1.4)$ \\
\hline Prior MI & $30(21.2)$ \\
\hline \multicolumn{2}{|l|}{ CAD type } \\
\hline Stable angina & $72(50.7)$ \\
\hline UA/NSTEMI & $1(0.7)$ \\
\hline Asymptomatic CAD & $69(48.6)$ \\
\hline
\end{tabular}

Data given as mean \pm SD or $n(\%)$. BMI, body mass index; CABG, coronary artery bypass grafting; $C A D$, coronary artery disease; MI, myocardial infarction; NSTEMI, non-ST-elevated myocardial infarction; $\mathrm{PCl}$, percutaneous coronary intervention; $\mathrm{UA}$, unstable angina.
The Youden index was used to identify the best cut-off for every index; the 3-D QCA-derived index and QFR were used for predicting functionally significant stenosis. All statistical analysis was performed using $\mathrm{JMP}^{\circledR} 12$ (SAS Institute, Cary, NC, USA).

\section{Results}

\section{Baseline Characteristics}

A total of 20 vessels, which accounted for $12 \%$ of all vessels, were excluded due to the aforementioned exclusion criteria for the selection of the 2 optimal CAG images. The remaining 151 vessels in 142 patients underwent QFR analysis. Patient and lesion characteristics are listed in Tables 1,2. Average $\mathrm{DS} \%$, lesion length, MLD, reference diameter, and $\mathrm{AS} \%$

\begin{tabular}{|c|c|}
\hline Lesion characteristics & \\
\hline \multicolumn{2}{|l|}{ Index artery } \\
\hline Left anterior descending artery & $96(63.6)$ \\
\hline Left circumflex artery & $25(16.6)$ \\
\hline Right coronary artery & $26(17.2)$ \\
\hline Diagonal artery & $2(1.3)$ \\
\hline Saphenous vein graft & $1(0.7)$ \\
\hline Left main trunk & $1(0.7)$ \\
\hline Bifurcation lesions & $71(47.0)$ \\
\hline Stented lesion & $21(13.9)$ \\
\hline Tandem/diffuse lesion & $47(31.1)$ \\
\hline \multicolumn{2}{|l|}{ Fractional flow reserve } \\
\hline Mean \pm SD & $0.84 \pm 0.08$ \\
\hline Median (IQR) & $0.85(0.79-0.92)$ \\
\hline Percent diameter stenosis & $48.8 \pm 8.2$ \\
\hline Percent area stenosis & $62.2 \pm 11.9$ \\
\hline Minimum lumen diameter (mm) & $1.38 \pm 0.39$ \\
\hline Reference vessel diameter (mm) & $2.84 \pm 0.57$ \\
\hline Lesion length (mm) & $16.8(12.1-24.6)$ \\
\hline \multicolumn{2}{|c|}{ Severity of CS (ACC/AHA classification) } \\
\hline $50 \%$ & $64(42.4)$ \\
\hline $75 \%$ & $87(57.6)$ \\
\hline
\end{tabular}

Data given as $\mathrm{n}(\%)$, mean $\pm \mathrm{SD}$, or median (IQR). Anatomical parameters were quantified on 3-dimensional quantitative coronary angiography. ACC/AHA, American College of Cardiology/American Heart Association; CS, coronary stenosis.
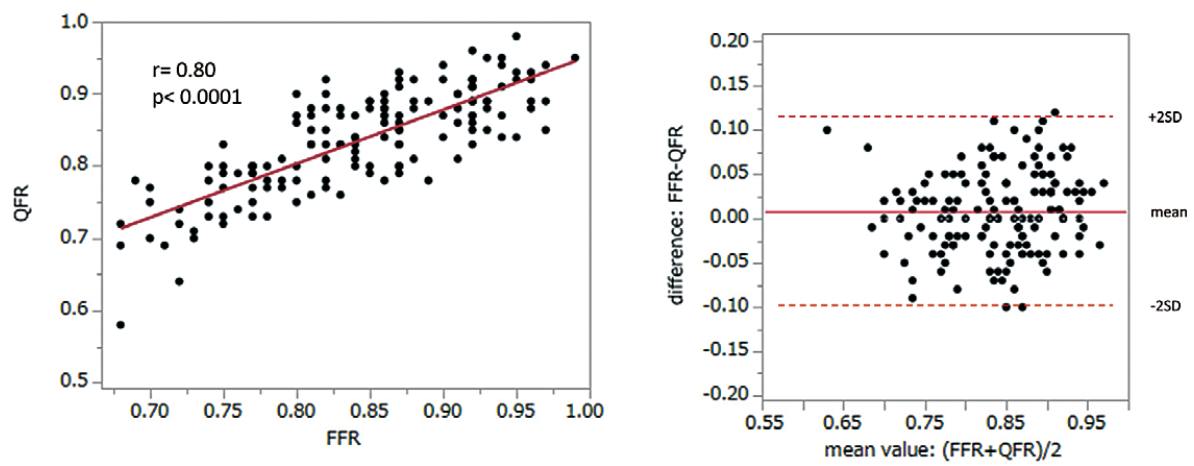

Figure 2. Correlation and agreement of quantitative flow ratio (QFR) with the conventional wire-based fractional flow reserve (FFR). 
of interrogated vessels were $48.8 \pm 8.2 \%, 19.79 \pm 10.65 \mathrm{~mm}$, $1.45 \pm 0.95 \mathrm{~mm}, 2.84 \pm 0.57 \mathrm{~mm}$, and $62.2 \pm 11.9 \%$, respectively. The measured FFR was $0.84 \pm 0.08$ and abnormal FFR $\leq 0.8$ was recorded for 46 vessels $(30.5 \%)$.

\section{Correlation With FFR}

Average QFR was $0.84 \pm 0.07$. QFR had good correlation $(\mathrm{r}=0.80, \mathrm{P}<0.0001)$ and agreement (mean difference, $0.01 \pm 0.05$ ) with FFR (Figure 2). In contrast, other anatomical indices such as AS $\%$, DS $\%$, and MLD had weaker correlations $(\rho=-0.26, \mathrm{P}=0.001 ; \rho=-0.37, \mathrm{P}<0.0001$; and $\rho=0.52, \mathrm{P}<0.0001)$ with FFR than QFR.

\section{Accuracy of QFR}

On ROC analysis with an FFR cut-off $\leq 0.8$, QFR had a greater AUC (0.93) than DS\% (AUC, 0.76), MLD (AUC, 0.78 ), and $\mathrm{AS} \%$ (AUC, 0.68; Figure 3). Applying the FFR cut-off $\leq 0.8$ to QFR $\leq 0.8$ resulted in 41 true-positive, 93 true-negative, 12 false-positive, and 5 false-negative results. The accuracy, sensitivity, specificity, PPV, and NPV of QFR were $88.7 \%, 89.1 \%, 88.6 \%, 77.4 \%$, and $94.9 \%$, respectively (Table 3).

\section{Calculation of QFR}

The average time to calculate QFR was 266s (IQR, 181$332 \mathrm{~s})$. This included time spent selecting 2 optimal angiographic images to complete the QFR calculation.

\section{Discussion}

\section{Main Findings}

QFR had good correlation $(\mathrm{r}=0.80, \mathrm{P}<0.0001)$ and agreement (mean difference: $0.01 \pm 0.05$ ) with wire-based FFR. When FFR $\leq 0.8$ was used as the cut-off for diagnosis of myocardial ischemia, QFR had larger AUC of 0.93 on ROC analysis than did 3-D QCA-derived anatomical indices. When the QFR cut-off was also set at 0.8 , it provided acceptable diagnostic performance (sensitivity, specificity, PPV, and NPV: $88.7 \%, 89.1 \%, 88.6 \%, 77.4 \%$, and $94.9 \%$ ). Moreover, QFR calculation required a relatively short time for the entire analysis.

\section{Previous Modalities}

CFD simulation has contributed to virtual FFR computation (e.g., CT-FFR, 3-D QCA-based FFR), as previously reported. ${ }^{9,11} \mathrm{CFD}$ plays an important role in reconstructing coronary trees and enables the recognition of functional severity in intermediate stenosis. Complex computation, however, requires a longer time to create a precise map of the coronary tree. Complete analysis of CT-FFR and calculation of rotational angiography-based FFR, based on CFD, take at least several hours despite the good diagnostic

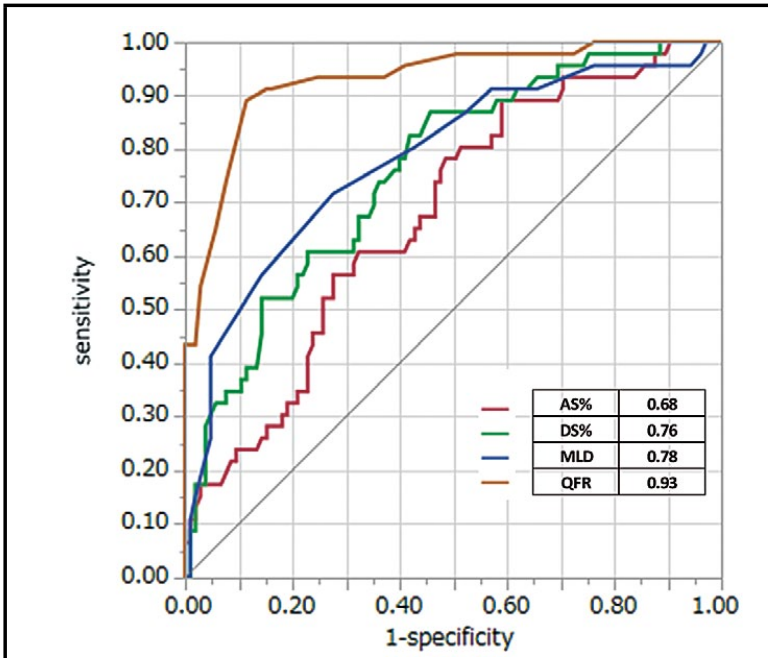

Figure 3. Receiver operating characteristics curve with fractional flow reserve cut-off $\leq 0.8$. Area under the curve was significantly greater for the quantitative flow ratio (QFR) computation model compared with the 3-dimensional anatomical indices. AS\%, percent area stenosis; DS\%, percent diameter stenosis; MLD, minimum lumen diameter.

performance. ${ }^{7,13} \mathrm{Tu}$ et al took $<10 \mathrm{~min}$ to calculate 3-D QCA-based FFR using the newly developed algorithm. ${ }^{11}$ We also achieved a shorter QFR analysis time of, at most, 590s (median, 266 s; IQR, 181-332s), which was an acceptable time to calculate values. Moreover, contrast-flow QFR (which is based on computational hyperemic state and TIMI frame count) used in the present study had good correlation and agreement with wire-based FFR and acceptable diagnostic performance. Tu et al reported that QFR based on the pharmacologically induced hyperemic state did not have better diagnostic performance than contrast-flow QFR, ${ }^{15}$ which might be explained by the following: (1) the contrast could induce submaximum hyperemia similar to the pharmacologically induced state; (2) CFV could be accelerated by pharmacological hyperemia, which could lead to underestimation of functional ischemia; and (3) deterioration of angiographic images induced by pharmacological hyperemia with increasing heart beat and flow velocity. We did not analyze QFR based on pharmacologic hyperemia in the present study; therefore, we could not compare contrast-flow QFR and QFR based on pharmacologic hyperemia. We were, however, able to determine an acceptable value of QFR with regard to diagnostic accuracy, correlation, and agreement with FFR, as Tu et al demonstrated, thereby demonstrating the feasibility of QFR.

\begin{tabular}{|c|c|c|c|c|}
\hline Diagnostic measure & QFR $\leq 0.8$ & DS $\% \geq 47.5 \%$ & AS $\% \geq 58 \%$ & MLD $\leq 1.20$ \\
\hline Diagnostic accuracy (\%) & 88.7 & 64.2 & 55.6 & 72.2 \\
\hline Sensitivity (\%) & 89.1 & 87.0 & 89.1 & 71.7 \\
\hline Specificity (\%) & 88.6 & 54.3 & 41.0 & 72.4 \\
\hline PPV (\%) & 77.4 & 40.5 & 39.8 & 53.2 \\
\hline NPV (\%) & 94.9 & 90.5 & 89.6 & 85.4 \\
\hline
\end{tabular}

AS\%, percent area stenosis; DS\%, percent diameter stenosis; FFR, fractional flow reserve; MLD, minimum lumen diameter; NPV, negative predictive value; PPV, positive predictive value; QCA, quantitative coronary angiography; QFR, quantitative flow ratio. 


\section{Clinical Implications of QFR}

QFR analysis required less evaluation time and, hence, could be used for online analysis. Furthermore, online QFR may have the potential to augment the information on individual functional severity in multiple intermediate coronary stenosis, which may be utilized in coronary revascularization. To utilize QFR for a more accurate clinical evaluation, we should take into account the wide variation in agreement between QFR and FFR at the approximate value of 0.8 . It is possible that the hybrid strategy might partially solve the problem based on subanalysis of the present cohort. When limited lesions with QFR $<0.75$ or $>0.85$ were examined $(n=85)$, the diagnostic performance of QFR for FFR $\leq 0.8$ was excellent, with diagnostic accuracy, sensitivity, specificity, PPV, and NPV of $96 \%, 84 \%$, $100 \%, 100 \%$, and $96 \%$, respectively. Using this proposed hybrid method, FFR should be measured in lesions with QFR 0.75-0.85. This may have the potential to be a sophisticated method of evaluation for myocardial ischemia using both QFR and FFR.

\section{Study Limitations}

This study was a retrospective, observational study involving a small sample size at a single center. Because of the retrospective analysis of CAG, we could not always obtain the appropriate images for reconstruction of 3-D QCA, which partially influenced patient selection. As a result, $12 \%$ of all vessels were excluded due to the absence of optimal angiography. Moreover, we could not validate the interobserver or intraobserver variability in these selected vessels. It is difficult to recommend the practical use of QFR for estimating myocardial ischemia due to the following reasons: (1) QFR could require more contrast agent to achieve optimal angiographic visualization; (2) it is unclear whether QFR can yield precise values independent of lesion characteristics or vessel characteristics; and (3) in the present study, only intermediate stenosis was included; therefore, we could not confirm that the outcome is independent of lesion severity. We enrolled various consecutive targets, including bifurcation and tandem/diffuse or stented lesions in the main epicardial artery, bypass graft, or side branches to exclude selection bias. This enrolling of consecutive targets suggests the potential benefit of QFR for lesions evaluated using only angiography, which may also be attractive for catheter interventionists.

\section{Conclusions}

QFR had good correlation and agreement with wire-based FFR and high diagnostic performance in the evaluation of intermediate coronary stenosis, suggesting its potential as an alternative tool for estimating myocardial ischemia.

\section{Acknowledgments}

We thank Editage (www.editage.jp) for English language editing.

\section{Funding}

None.

\section{Disclosures}

The authors declare no conflict of interest.

\section{References}

1. Kern MJ, Samady H. Current concepts of integrated coronary physiology in the catheterization laboratory. J Am Coll Cardiol
2010; 55: 173-185.

2. Tonino PAL, De Bruyne B, Pijls NHJ, Siebert U, Ikeno F, van't Veer M, et al. Fractional flow reserve versus angiography for guiding percutaneous coronary intervention. N Engl J Med 2009; 360: $213-224$.

3. van Nunen LX, Zimmermann FM, Tonino PAL, Barbato E, Baumbach A, Engstrøm T, et al. Fractional flow reserve versus angiography for guidance of PCI in patients with multivessel coronary artery disease (FAME): 5-year follow-up of a randomised controlled trial. Lancet 2015; 386: 1853-1860.

4. Fihn SD, Blankenship JC, Alexander KP, Bittl JA, Byrne JG, Fletcher BJ, et al. 2014 ACC/AHA/AATS/PCNA/SCAI/STS focused update of the guideline for the diagnosis and management of patients with stable ischemic heart disease: A report of the American College of Cardiology/American Heart Association Task Force on Practice Guidelines, and the American Association for Thoracic Surgery, Preventive Cardiovascular Nurses Association, Society for Cardiovascular Angiography and Interventions, and Society of Thoracic Surgeons. J Am Coll Cardiol 2014; 64: $1929-1949$.

5. Pijls NH, van Schaardenburgh P, Manoharan G, Boersma E, Bech JW, van't Veer M, et al. Percutaneous coronary intervention of functionally nonsignificant stenosis: 5 -year follow-up of the DEFER Study. J Am Coll Cardiol 2007; 49: 2105-2111.

6. Pijls NHJ. Fractional flow reserve to guide coronary revascularization. Circ J 2013; 77: 561-569.

7. Morris PD, Ryan D, Morton AC, Lycett R, Lawford PV, Hose $\mathrm{DR}$, et al. Virtual fractional flow reserve from coronary angiography: Modeling the significance of coronary lesions: Results from the VIRTU-1 (VIRTUal Fractional Flow Reserve From Coronary Angiography) study. JACC Cardiovasc Interv 2013; 6: $149-157$.

8. Morris PD, van de Vosse FN, Lawford PV, Hose DR, Gunn JP. "Virtual" (computed) fractional flow reserve: Current challenges and limitations. JACC Cardiovasc Interv 2015; 8: 1009-1017.

9. Norgaard BL, Leipsic J, Gaur S, Seneviratne S, Ko BS, Ito H, et al. Diagnostic performance of noninvasive fractional flow reserve derived from coronary computed tomography angiography in suspected coronary artery disease: The NXT trial (Analysis of Coronary Blood Flow Using CT Angiography: Next Steps). $J$ Am Coll Cardiol 2014; 63: 1145-1155.

10. Papafaklis MI, Muramatsu T, Ishibashi Y, Lakkas LS, Nakatani $\mathrm{S}$, Bourantas CV, et al. Fast virtual functional assessment of intermediate coronary lesions using routine angiographic data and blood flow simulation in humans: Comparison with pressure wire: Fractional flow reserve. EuroIntervention 2014; 10: 574-583.

11. Tu S, Barbato E, Koszegi Z, Yang J, Sun Z, Holm NR, et al. Fractional flow reserve calculation from 3-dimensional quantitative coronary angiography and TIMI frame count: A fast computer model to quantify the functional significance of moderately obstructed coronary arteries. JACC Cardiovasc Interv 2014; 7: 768-777.

12. Koo BK, Erglis A, Doh JH, Daniels DV, Jegere S, Kim HS, et al. Diagnosis of ischemia-causing coronary stenoses by noninvasive fractional flow reserve computed from coronary computed tomographic angiograms. Results from the prospective multicenter DISCOVER-FLOW (Diagnosis of Ischemia-Causing Stenoses Obtained Via Noninvasive Fractional Flow Reserve) study. J Am Coll Cardiol 2011; 58: 1989-1997.

13. Min JK, Leipsic J, Pencina MJ, Berman DS, Koo BK, van Mieghem C, et al. Diagnostic accuracy of fractional flow reserve from anatomic CT angiography. JAMA 2012; 308: 1237-1245.

14. Nakazato R, Park HB, Berman DS, Gransar H, Koo BK, Erglis A, et al. Noninvasive fractional flow reserve derived from computed tomography angiography for coronary lesions of intermediate stenosis severity: Results from the DeFACTO study. Circ Cardiovasc Imaging 2013; 6: 881-889.

15. Tu S, Westra J, Yang J, von Birgelen C, Ferrara A, Pellicano M, et al. Diagnostic accuracy of fast computational approaches to derive fractional flow reserve from diagnostic coronary angiography: The International Multicenter FAVOR Pilot Study. JACC Cardiovasc Interv 2016; 9: 2024-2035.

16. De Bruyne B, Paulus WJ, Pijls NHJ. Rationale and application of coronary transstenotic pressure gradient measurements. Cathet Cardiovasc Diagn 1994; 33: 250-261.

17. Kirkeeide RL. Coronary obstructions, morphology and physiologic significance. In: Reiber JHC, Serruys PW, editors. Quantitative coronary arteriography. Dordrecht: Springer Netherlands, $1991 ; 229-244$. 